\section{OPEN ACCESS}

Edited by:

Miguel-Angel Gomez-Ruano, Polytechnic University of Madrid,

Spain

Reviewed by:

Jose Maria Gonzalez-Rave, University of Castilla La Mancha,

Spain

Helmi Chaabene,

Universität Potsdam, Germany

${ }^{*}$ Correspondence:

Javier Sanchez-Sanchez jsanchezsa@upsa.es

Specialty section: This article was submitted to Movement Science and Sport

Psychology,

a section of the journal

Frontiers in Psychology

Received: 21 January 2019

Accepted: 03 May 2019

Published: 06 June 2019

Citation:

Petisco C, Ramirez-Campillo $R$, Hernández D, Gonzalo-Skok O,

Nakamura FY and

Sanchez-Sanchez J (2019)

Post-activation Potentiation: Effects of Different Conditioning Intensities on Measures of Physical Fitness in Male

Young Professional Soccer Players.

Front. Psychol. 10:1167.

doi: 10.3389/fpsyg.2019.01167

\title{
Post-activation Potentiation: Effects of Different Conditioning Intensities on Measures of Physical Fitness in Male Young Professional Soccer Players
}

\section{Cristina Petisco', Rodrigo Ramirez-Campillo 1,2, Daniel Hernández', Oliver Gonzalo-Skok ${ }^{1,3}$, Fabio Y. Nakamura ${ }^{1,4,5}$ and Javier Sanchez-Sanchez ${ }^{1 *}$}

'Research Group Planning and Assessment of Training and Athletic Performance, Pontifical University of Salamanca, Salamanca, Spain, ${ }^{2}$ Laboratory of Human Performance, Research Nucleus in Health, Physical Activity and Sport, Department of Physical Activity Sciences, Universidad de Los Lagos, Osorno, Chile, ${ }^{3}$ Faculty of Health Sciences, University of San Jorge, Zaragoza, Spain, ${ }^{4}$ College of Healthcare Sciences, James Cook University, Townsville, QLD, Australia, ${ }^{5}$ Department of Medicine and Aging Sciences, "G. d'Annunzio" University of Chieti-Pescara, Chieti, Italy

The aim of this study was to compare the effects of different warm-up conditioning intensities on the physical fitness (i.e., post-activation potentiation -PAP), of professional male field soccer players. Athletes ( $n=10$; age: $21.6 \pm 3.2$ years) completed a control warm-up and warm-ups aimed to induce PAP, in random and counterbalanced order. After control and experimental warm-up sessions participants completed a triple hop test with the dominant (H3Jd) and a non-dominant (H3Jnd) leg, a squat jump (SJ), a countermovement jump (CMJ), a change of direction ability (COD) test, a repeated sprint with a COD (RSCOD) test and a linear 30-m sprint test (S-30). The control warmup (WU) protocol was designed according to athlete's regular warm-up practice. The experimental warm-ups included the same exercises as the WU, with addition of one set of half-back squats for 10 repetitions at $60 \%, 5$ repetitions at $80 \%$, and 1 repetition at $100 \%$ of $1 \mathrm{RM}$ (60\%-1RM, 80\%-1RM and 100\%-1RM, respectively.) Threshold values for Cohen's effect sizes (ES) were calculated and used for group's comparison. Likely to most likely improvements were shown in H3Jd ( $E S=0.52$ ), H3Jnd ( $E S=0.51$ ), COD ( $E S=0.38)$, fasted sprint $(\mathrm{RSCODb})(E S=0.58)$ and the total time of all sprints (RSCODt) $(E S=0.99)$ only after the $80 \%-1 \mathrm{RM}$ protocol in comparison to the WU. Conversely, 100\%-1RM and 60\%-1RM protocols, compared to WU, induced possibly to most likely poorer performance in all jumps, COD and RSCODb ( $E S=-0.07$ to -1.03 and $E S=-0.48$ to -0.91 , respectively). Possibly to most likely improvements were shown in all jumps, COD, RSCODb and RSCODt after the 80\%-1RM warm-up protocol in comparison to the 100\%-1RM and 60\%-1RM warm-up protocols ( $E S=0.35$ to 2.15 and $E S=0.61$ to 1.46 , respectively). A moderate warm-up intensity (i.e., 80\%-1RM back squat) may induce greater PAP, including improvements in jumping, repeated and non-repeated change of direction speed in male soccer players.

\footnotetext{
Keywords: plyometric, speed, fatigue, warm-up, soccer
} 


\section{INTRODUCTION}

Aside from the total distance covered, performing highintensity actions repeatedly during a match is a key feature of soccer (Stølen et al., 2005). During a competitive match, soccer players may perform $\sim 1400$ short-duration maximal or near maximal intensity activities, including sprints, change of directions (COD), tackling, accelerations, decelerations, jumps, among others (Iaia et al., 2009). Although training programs may improve such performance actions at long-term, shortterm (or acute) improvements may also be induced by warmup activities, a method routinely used by athletes, coaches and strength and conditioning specialists to improve muscle force and power involved in athletic performance during competition (Evetovich et al., 2015).

A large amount of research regarding the effects of a warmup on human performance have been conducted (Rahimi, 2014; Barnes et al., 2015; González-Mohíno et al., 2018). There is a general consensus pointing the benefits of a warm-up on subsequent performance (Lockie et al., 2017). However, the optimal warm-up strategy for soccer players before a match is not well established (Hammami et al., 2018). Most soccer-related warm-up strategies involved static and dynamic stretching, neuromuscular activities, and short-duration high-intensity activities (Zois et al., 2011). Regarding the latter, they may induce post-activation potentiation (PAP) (Evetovich et al., 2015).

The PAP is a phenomenon in which neuromuscular performance characteristics are enhanced after intense contractile stimulation (Hodgson et al., 2005). The PAP in humans may be induced by isometric maximum voluntary contraction (Hamada et al., 2000), high-intensity resistance stimulus (McBride et al., 2005), and plyometric exercise (Turner et al., 2015). Although the existing research tends to reveal inconsistent findings (Moir et al., 2009), some studies has shown that performing muscular contractions under near-maximal load conditions improves subsequent performance during movements requiring large muscular power outputs of the stimulated muscle groups (Hodgson et al., 2005). However, there is little research regarding the effects of heavy resistance exercise on subsequent performance in soccer (Hammami et al., 2018). Some authors indicated that heavy resistance exercise may improve subsequent jump and COD (Zois et al., 2011), repeated sprints (Low et al., 2015; Sanchez-Sanchez et al., 2018) and single linear sprint (McBride et al., 2005; Tillin and Cooke, 2009).

The exact mechanism responsible for this PAP response remains uncertain (Lockie et al., 2017). Chemical, neuromuscular and mechanical changes may occur that temporarily aid the contractile properties of muscle tissue (Sale, 2004). One common PAP mechanism theory indicates the phosphorylation of myosin light chains resulting from the initial muscle activity, which would turn the actin and myosin molecules more sensitive to calcium (Ca2+) availability (Tillin and Bishop, 2009). Also it is speculated that PAP can increase the excitability of motoneurons (Hodgson et al., 2005) and enhancement of neural output by recruitment of faster motor units (Hamada et al., 2000; Sale, 2004). Moreover, it was also reported that pre-loading may increase muscle stiffness (Moir et al., 2009). Previous studies have suggested that PAP responses might be dependent on individual characteristics (Sanchez-Sanchez et al., 2018). For instance, stronger subjects exhibited a greater PAP response when compared with weaker counterparts (Seitz and Haff, 2016). In contrast, other studies concluded that performance after a PAP inducing activity was not related to training status (McBride et al., 2005). Furthermore, it has been proposed that the PAP may be related to the type of muscle fiber being activated (Wilson et al., 2013), with a higher proportion of fast fibers being related to greater PAP effect (Sole et al., 2013).

Therefore, in order to maximize PAP, these factors (e.g., training status) should be taken into account together with the load used during the warm-up (Lockie et al., 2017). McBride et al. (2005), for example, demonstrated that 1 set of 3 repetitions at $90 \%$ of one repetition maximum (1RM) significantly improve sprint time performance, whilst 1 set of 3 repetitions at $30 \%$ $1 \mathrm{RM}$ did not. In the same context, a previous study conducted with male soccer players showed that in order to induce optimal running speed enhancements, it is necessary to set the intensity of the warm-up protocol with loads $\geq 80 \%$-1RM (Rahimi, 2014).

The competition rules require the physical trainers to finish the warm-up 15-20 min before the start to the matches. The PAP could be used between the end of the warm up and the start of the game, to maintain the level of activation in the players (Russell et al., 2014). Although greater loads have been recommended to induce PAP in strength and power tasks (McBride et al., 2005; Rahimi, 2014), its practical application in soccer is difficult. This is because of the limited time-frame separating the end of the warm-up and the start of a soccer match which is not sufficient to include multiple sets (Russell et al., 2014). Therefore, the aim of this study was to compare the effects of different warm-up conditioning intensities on physical fitness (i.e., PAP), of professional male field soccer players.

\section{MATERIALS AND METHODS}

\section{Participants}

Professional male soccer players $(n=10$; age: $21.6 \pm 3.2$ years, body height: $177.9 \pm 4.3 \mathrm{~cm}$, and body mass: $69.5 \pm 3.1 \mathrm{~kg}$ ) with $\geq 6$-years of training and competition experience were recruited for the study. Their regular training schedule involved four training sessions plus a competitive match per week in the Spanish second division "B." All participants: (1) were field players (four defenders, four midfielders and two forward), (2) were free of injuries in the last 3-months, (3) had regularly trained and competed in the past 6-months and (4) haven't got any lower extremity surgery in the past 2-years. Soccer players signed an informed consent before starting the data collection. The protocol was approved by the Ethics Committee of the Pontifical University of Salamanca (Annex III, Act 13/2/2019) and conformed to the latest version of the Declaration of Helsinki.

\section{Procedures}

The experiments were conducted during the competitive period of the season 2018. The control and experimental warm-up sessions were completed in a random, counterbalanced order, 
completed in a period of 3-weeks. The tests during control and experimental sessions were completed in the same order, between 15:00 and 20:00 h, at an indoor venue, with the same sports clothes and by the same investigator, who was blinded to the group allocation of the participants. To avoid the effects of fatigue on testing results, participants completed the control and the experimental warm-up sessions no less than $48 \mathrm{~h}$ after the last training/competition session. Each intervention was applied twice, making the tests in the following order. After control and experimental warm-up sessions participants completed measure in day 1: triple hop test with dominant (H3Jd) and non-dominant (H3Jnd) leg, squat jump (SJ) and repeated sprint with COD (RSCOD); measure in day 2: linear 30-m sprint test (S-30), countermovement jump (CMJ), COD test. The recovery between tests was $1 \mathrm{~min}$. Participants were asked to attend each session under an adequate feeding and hydration state. The testing protocols were performed in the facilities where athletes usually train and compete.

\section{Familiarization and Maximal Dynamic Strength Test}

During a 120 min familiarization session, athletes simulated the warm-up protocols and completed a maximal dynamic strength test (1RM) in order to assess the specific loads to be used during PAP warm-up sessions. The maximal dynamic strength was assessed through the half back squat exercise using the Smith machine (MultipowerPeroga ${ }^{\circledR}$, Murcia, Spain), with the barbell constrained to move along the vertical axis. The 1RM test was preceded by a 5-minutes low-intensity run in which the heart rate not exceed at $140 \mathrm{~b} \cdot \mathrm{min}^{-1}$ (Polar RS800CX, Electro Oy, Kempele, Finland), and by 5 and 3 half back squat repetitions at an estimated $50 \%$ and $70 \% 1 \mathrm{RM}$, respectively. In the initial position the barbell was at shoulder-level, feet at shoulder-width distance, and knee and hips in full extension. Adhesive marks were added to the floor and the barbell to assure consistency in the hands and feet position during testing. In addition, a wooden seat with adjustable heights was placed behind the subjects to keep bar displacement and knee angle $\left(\sim 90^{\circ}\right.$ knee angle $)$ constant on each half back squat attempt. The $1 \mathrm{RM}$ load was defined as the maximum weight that could be lifted once using the proper exercise technique through a full range of motion (Okuno et al., 2013). A 3-minutes rest interval was adopted between attempts, and the subjects had up to five attempts to obtain their 1RM.

\section{Jumping Test}

Athletes completed the H3Jd and H3Jnd (Noyes et al., 1991). Participants take maximal jumps forward as far as possible on the testing leg and land on two legs during the final jump. At the end of each horizontal jump attempt, athletes maintained the landing position for a brief moment. Soccer players also completed the CMJ and SJ tests following previous suggestions (Maulder et al., 2015), with minimal flexion of the trunk during take-off. Jumping was measured with a contact mat (Globus Ergo System $^{\circledR}$, Codogné, Italy). In all jumps, the hands were used freely, except during the SJ and CMJ, where athletes positioned arms akimbo. Athletes performed two maximal trials for each test with 1-minute of rest in between. The best value achieved was selected for analysis.

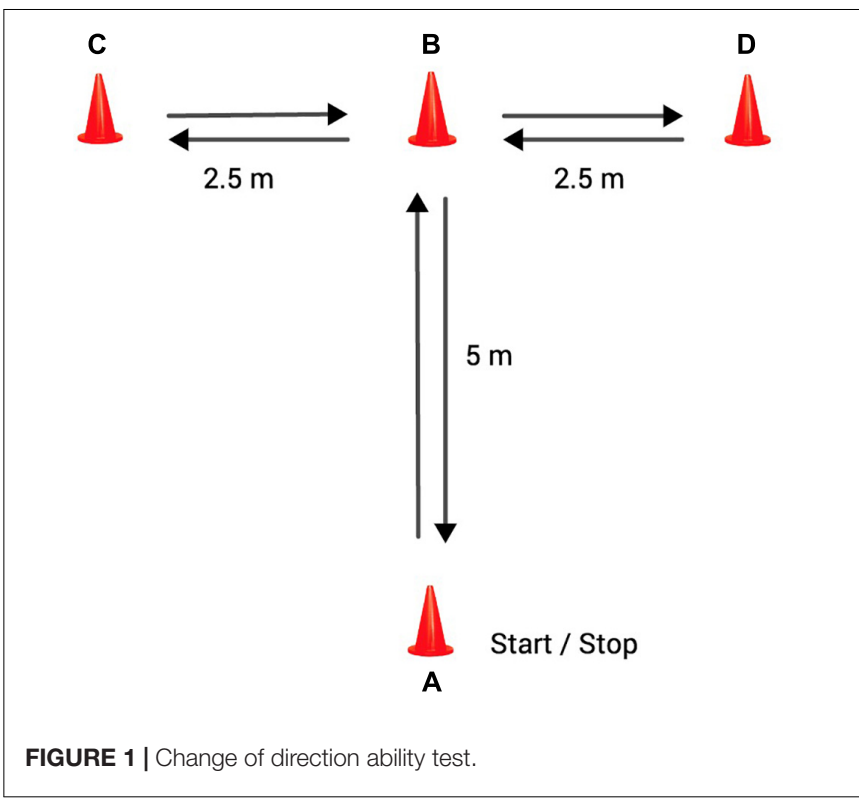

\section{Change of Direction Ability Test}

Athletes also completed a modified $t$-test (Sassi et al., 2009) to evaluate COD. A photocell gate system (Witty, Microgate ${ }^{\circledR}$, Italy) was used to record the time. The players performed the test using the same directives as the traditional test, although they were not required to move laterally or face forward (Figure 1). The players had to touch the top of the cones instead of its base. The displacement followed this route: $\mathrm{AB}$ displacement, at his own discretion, each subject sprinted forward to cone B and touched the top of the cone with the right hand; BC displacement, facing forward the participant shuffled to the left to cone $\mathrm{C}$ and touched the top of the cone with the left hand; CD displacement, the soccer player then shuffled to the right to cone D and touched its top; DB displacement, the players shuffled back to the left to cone B and touched its top; BA displacement, the players moved backward as quickly as possible and returned to line A. Players performed two maximal trials, with 1-minute of rest in between. The best value achieved was selected for analysis.

\section{Linear 30-m Sprint Test}

For maximal sprinting physical fitness assessment, athletes completed a S-30 sprint. The S-30 involved single maximaleffort sprints from a stationary start. Players initiated the sprint at their own discretion, positioning the foot $0.5-\mathrm{m}$ behind the start line. Thirty-meter linear sprint performance was assessed using a double-beam photocell system (Witty, Microgate ${ }^{\circledR}$, Italy). Athletes performed two maximal trials, with 1-minute of rest in between. The best value achieved was selected for analysis.

\section{Repeated Sprint With COD Test}

In addition to the linear sprint, athletes completed a RSCOD test. The RSCOD test included 6 sprints, with a passive recovery period of 20-seconds in between (Okuno et al., 2013). Each sprint involved $15-\mathrm{m}$ of linear sprint, a COD of $180^{\circ}$, and another $15-\mathrm{m}$ linear sprint $\left[15-\mathrm{m}+15-\mathrm{m}\left(\mathrm{COD}-180^{\circ}\right)\right]$. Times were recorded 


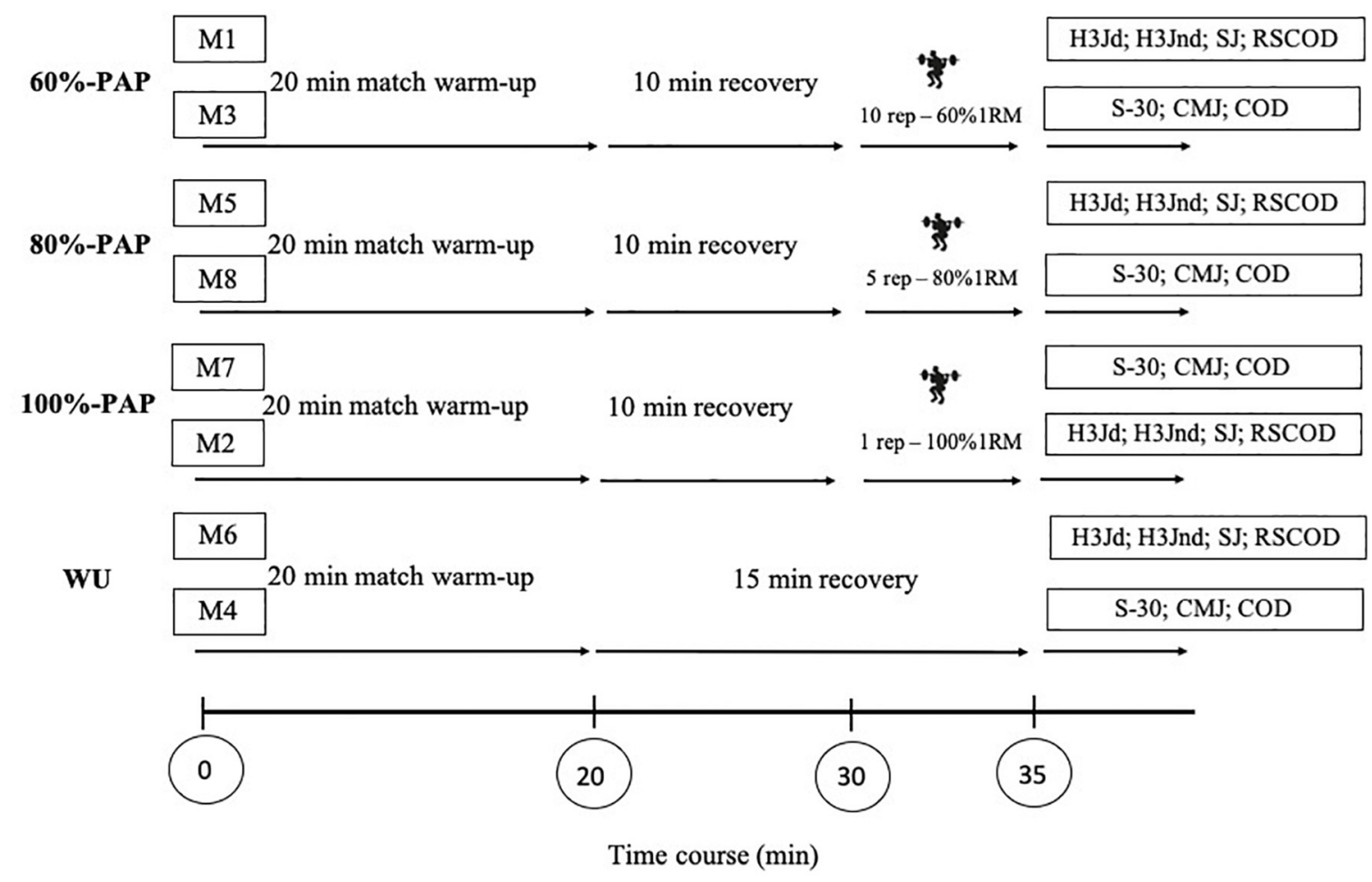

FIGURE 2 | Overview of the cross-over experimental design, indicating the control and three different warm-up conditions. The 15-minutes recovery period was included during control condition. The 10-minutes plus 5-minutes recovery period were included during PAP protocols, to simulate match conditions. The tests were divided at two measurement $(\mathrm{M})$ day corresponding to each protocols. 60\%-1RM, 80\%-1RM, and 100\%-1RM refer to match warm-up post-activation potentiation (PAP) protocols at 60, 80 and 100\% of 1RM, respectively; WU, match warm-up protocol; M, measurement; H3Jd, triple hop test with dominant leg; H3Jnd, triple hop test with non-dominant leg; SJ, squat jump test; RSCOD, repeated sprint with change of direction test; S-30, linear 30-m sprint test; CMJ, countermovement jump test; COD, change of direction ability test.

with a double-beam photocell system (Witty, Microgate ${ }^{\circledR}$, Italy). Athletes received verbal encouragement during the test. The players were verbally and visually informed to assume the starting position $0.5-\mathrm{m}$ behind the starting line 6-seconds before each sprint. Also a 3-seconds countdown was visually provided with a light panel (Microgate ${ }^{\circledR}$, Italy) that informed of the start of the next sprint. The fastest sprint (RSCODb) and the total time of all sprints (RSCODt) were retained for further analyses.

\section{Warm-Up Protocols}

The overview of warm-up protocols is presented in Figure 2. A control warm-up protocol (WU) was designed according to athlete's regular warm-up practices. The WU included 7minutes of general warm-up [i.e., continuous moderate-intensity $\left(\leq 10 \mathrm{~km} / \mathrm{h}^{-1}\right)$ running; general main-joint movements (mobility of upper and lower extremities; proprioceptive drills [landings with dominant and non-dominant leg after low-intensity frontal and lateral jumps)], 3-minutes of specific warm-up [i.e., elasticbands resisted drills and ballistic drills (lateral and frontal movements on 5-m and simulations of ball shots wearing an elastic on the ankles or knees)], 5-minutes of ball drills (i.e., basic technical drills with a partner), and 5-minutes of small-sided games (i.e., $5 \mathrm{vs5}$ in a $30 \times 20$-m size pitch). After the WU athletes rested passively for 15-minutes before testing, as it happens in a competition match. The experimental PAP warm-ups included the same exercises as the WU, with the addition (10-minutes after the end of WU) of one set of half back squats at 60,80 , or $100 \%$ of $1 \mathrm{RM}$ for a total of 10 repetitions (60\%-1RM), 5 repetitions $(80 \%$ $1 \mathrm{RM})$, or 1 repetition (100\%-1RM), respectively. All repetitions were performed at maximal voluntary concentric velocity. After each PAP load, athletes rested passively for 5-minutes before the H3JD, H3Jnd (measure in day 1) and S-30 tests (measure in day 2), 6-minutes before the SJ (measure in day 1) and CMJ tests (measure in day 2), and 8-minutes before the RSCOD (measure in day 1 ) and COD (measure in day 2 ), respectively.

\section{Statistical Analyses}

Data is presented as mean \pm standard deviation (SD). All data were first log-transformed to reduce biases arising from non-uniformity error. The standardized difference or effect size [ES, 90\% confidence limits (CL)] in the selected variables was calculated. Threshold values for Cohen's ES statistics were $>0.2$ (small), >0.6 (moderate), and > 1.2 (large) (Hopkins et al., 2009). For within-group comparisons, the chances that the differences in performance were better/greater [i.e., greater than the smallest worthwhile change ( 0.2 multiplied by the between-subject standard deviation, based on Cohen's $d$ principle)], similar, or worse/smaller were calculated. Quantitative chances (QC) of 
beneficial/better, similar/trivial, or detrimental/poorer effect were assessed qualitatively as follows: $<1 \%$, almost certainly not; $>1-5 \%$, very unlikely; $>5-25 \%$, unlikely; $>25-75 \%$, possible; $>75-95 \%$, likely; >95-99\%, very likely; and >99\%, most likely (Hopkins et al., 2009). If the chance that the true value is $>25 \%$ beneficial and $>0.5 \%$ chance that it is harmful, the clinically effect was considered as unclear. However, the clinical inference was declared as beneficial when odds ratio of benefit/harm was $>66 \%$ (Hopkins et al., 2009). A specific Excel spreadsheet from sportsci.org was used to examine the within-group (between PAP protocols) (xPostOnlyCrossover.xls) comparisons.

\section{RESULTS}

The mean 1RM half-squat for this study was $114.3 \pm 15.0 \mathrm{~kg}$. Table 1 shows the results for each test after each warmup protocol.

Comparisons between the control warm-up and the PAP warm-ups are indicated in Table 2 . Likely to most likely improvements were shown in $\mathrm{H} 3 \mathrm{Jd}, \mathrm{H} 3 \mathrm{Jnd}$, COD, RSCODb, and RSCODt only after the $80 \%-1 \mathrm{RM}$ protocol in comparison to WU. Furthermore, a possibly greater enhancement was found in RSCODt after the 60\%-1RM protocol compared to WU. Conversely, 100\%-1RM and 60\%-1RM protocols induced possible to most likely poorer performance in the H3Jd, H3Jnd, SJ, CMJ, COD, and RSCODb in comparison to WU. Similarly, a likely poorer S-30 performance was observed after $100 \%$ 1RM compared to WU.

Comparisons between the PAP warm-up protocols are indicated in Table 3. Possibly to most likely improvements were shown in H3Jd, H3Jnd, SJ, CMJ, COD, RSCODb, and RSCODt after the $80 \%$-1RM warm-up protocol in comparison to the $100 \%-1 \mathrm{RM}$ and 60\%-1RM warm-up protocols. In addition, a possible better performance was achieved in the S-30 after the $80 \%-1 \mathrm{RM}$ warm-up compared to the $100 \%$-1RM warm-up, while the $60 \%-1 \mathrm{RM}$ warm-up induced possible and very likely better S30 performance in comparison to the $80 \%-1 \mathrm{RM}$ and $100 \%-1 \mathrm{RM}$

TABLE 1| Physical performance of soccer players after different warm-up protocols.

\begin{tabular}{lllll}
\hline & WU & $\mathbf{1 0 0 \% - 1 R M}$ & $\mathbf{8 0 \% - 1 R M}$ & $\mathbf{6 0 \% - 1 R M}$ \\
\hline H3Jd (m) & $6.53 \pm 0.34$ & $6.40 \pm 0.36$ & $6.73 \pm 0.41$ & $6.26 \pm 0.49$ \\
H3Jnd (m) & $6.57 \pm 0.26$ & $6.56 \pm 0.43$ & $6.71 \pm 0.36$ & $6.32 \pm 0.39$ \\
SJ (cm) & $37.4 \pm 4.9$ & $36.7 \pm 4.9$ & $38.8 \pm 4.7$ & $34.5 \pm 5.4$ \\
CMJ (cm) & $39.8 \pm 3.2$ & $38.6 \pm 5.3$ & $40.7 \pm 4.7$ & $38.4 \pm 3.2$ \\
COD (s) & $7.23 \pm 0.27$ & $7.39 \pm 0.38$ & $7.12 \pm 0.26$ & $7.38 \pm 0.33$ \\
RSCODb (s) & $5.77 \pm 0.15$ & $5.94 \pm 0.11$ & $5.67 \pm 0.13$ & $5.89 \pm 0.21$ \\
RSCODt (s) & $35.7 \pm 0.65$ & $35.8 \pm 0.62$ & $35.0 \pm 0.67$ & $35.5 \pm 0.70$ \\
S-30 (s) & $4.05 \pm 0.23$ & $4.13 \pm 0.23$ & $4.09 \pm 0.28$ & $4.04 \pm 0.23$
\end{tabular}

H3Jd and H3Jnd, triple hop test with dominant and non-dominant leg, respectively, SJ, squat jump; CMJ, countermovement jump; COD, change of direction ability test; RSCODb and RSCODt, repeated sprint with change of direction best and total times, respectively, S-30, linear 30-m sprint test. WU, control warm-up protocol; 100\%-1RM, 80\%-1RM, and 60\%-1RM, PAP warm-up protocols at 60, 80, and $100 \%$ of one repetition maximum in half-squat. warm-up protocols, respectively. Finally, better performance in H3Jd (possible), H3Jnd (likely) and SJ (likely) were observed after $100 \%-1$ RM warm-up compared to $60 \%$-1RM warm-up, whereas better performance in RSCODb (possible), RSCODt (likely), and S-30 (very likely) were observed after the 60\%-1RM warm-up compared to the $100 \%-1$ RM warm-up.

\section{DISCUSSION}

The aim of this study was to compare the effects of different conditioning intensities on the physical fitness (i.e., PAP) of professional male field soccer players. Main results indicate improvements in jumping, single and repeated COD speed after the $80 \%-1 \mathrm{RM}$ protocol in comparison to WU. Moreover, better jumping, as well as single and repeated COD speed improvements were observed after the $80 \%-1 \mathrm{RM}$ compared to the $60 \%-1 \mathrm{RM}$ and $100 \%-1 \mathrm{RM}$ protocols. Therefore, a moderate intensity (i.e., $80 \%-1 \mathrm{RM}$ ) appears to be more effective than low (i.e., 60\%-1RM) and maximal (i.e., 100\%-1RM) warm-up strategies to induce greater PAP, including greater jumping, single and repeated COD speed in male soccer players.

Regarding jumping performance, likely to most likely improvements were shown in H3Jd and H3Jnd only after the $80 \%-1 \mathrm{RM}$ protocol in comparison to WU. Moreover, possibly to most likely improvements were shown in H3Jd, H3Jnd, SJ, and CMJ test after the $80 \%-1 \mathrm{RM}$ warm-up protocol in comparison to the 100\%-1RM and 60\%-1RM warm-up protocols. Improvements in jumping performance after loaded squat PAP protocols have been previously observed in male athletes from team-sports such as rugby, volleyball and soccer (Gouvêa et al., 2013), and may be explained by several neuro-mechanical shortterm adaptations (e.g., increased muscle-tendon stiffness) (Tillin and Cooke, 2009). Moreover, the PAP effects depend on the balance between fatigue and neuromuscular potentiation (Tillin and Bishop, 2009), which in turn depends on the load-related intensity used (Sale, 2004). In the current study, a load of moderate-intensity (i.e., $80 \%-1 \mathrm{RM}$ ) induced greater jumping performance improvements compared with loads of lower (i.e., $60 \%-1 \mathrm{RM}$ ) or greater (i.e., 100\%-1RM) intensity, agreeing with previous studies that found greater PAP effects after loads of intermediate intensity (Gouvêa et al., 2013). Of note, the greater PAP effect after intermediate-intensity loads may be particularly important when PAP actions are performed with the intention of maximizing movement velocity, leading to the recruitment of fast-twitch muscle fibers, which is considered a key factor to induce PAP (Turner et al., 2015). This improvement in jumping performance after loaded squats have been observed even after $6 \mathrm{~h}$ from the PAP warm-up (Saez-Saez de Villarreal et al., 2007). In addition, is important to note that soccer players' characteristics may affect the PAP magnitude (SanchezSanchez et al., 2018), thus current results should be interpreted considering the high training level of the soccer players.

The results indicate likely improvements in the COD after the $80 \%-1 \mathrm{RM}$ protocol in comparison to $\mathrm{WU}$, and likely poorer performance after the 100\%-1RM and 60\%-1RM protocols. Current outcomes are difficult to compare with previous findings, 
TABLE 2 | Comparisons of soccer player's physical performance after a traditional (control) warm-up versus three different warm-up conditions.

\begin{tabular}{|c|c|c|c|c|}
\hline & $\%$ (CL90\%) & ES (CL90\%) & Chances & Outcome \\
\hline \multicolumn{5}{|c|}{ WU vs. $100 \%-1$ RM } \\
\hline H3Jd & $-2.0(-4.4 ; 0.5)$ & $-0.35(-0.79 ; 0.08)$ & $2 / 24 / 73 \%$ & Possibly \\
\hline H3Jnd & $-0.3(-2.7 ; 2.2)$ & $-0.07(-0.65 ; 0.52)$ & $21 / 44 / 34 \%$ & Possibly \\
\hline SJ & $-2.0(-8.1 ; 4.5)$ & $-0.15(-0.61 ; 0.32)$ & $10 / 48 / 42 \%$ & Possibly \\
\hline CMJ & $-3.5(-8.7 ; 1.9)$ & $-0.41(-1.04 ; 0.21)$ & $5 / 22 / 73 \%$ & Possibly \\
\hline COD & $-2.1(-4.4 ; 0.2)$ & $-0.50(-1.04 ; 0.04)$ & $2 / 14 / 83 \%$ & Likely \\
\hline RSCODb & $-3.0(-3.9 ;-2.1)$ & $-1.03(-1.32 ;-0.73)$ & 0/0/100\% & Most Likely \\
\hline RSCODt & $-0.3(-0.5 ;-0.1)$ & $-0.14(-0.25 ;-0.03)$ & 0/83/17\% & Likely trivial \\
\hline S-30 & $-1.7(-2.2 ;-1.2)$ & $-0.28(-0.36 ;-0.20)$ & 0/5/95\% & Likely \\
\hline \multicolumn{5}{|c|}{ WU vs. $80 \%-1 R M$} \\
\hline H3Jd & $3.0(0.7 ; 5.3)$ & $0.52(0.13 ; 0.91)$ & $92 / 8 / 0 \%$ & Likely \\
\hline H3Jnd & $2.2(0.2 ; 4.2)$ & 0.51 (0.05; 0.97) & $88 / 11 / 1 \%$ & Likely \\
\hline SJ & $3.8(-3.0 ; 11.1)$ & $0.27(-0.22 ; 0.76)$ & $60 / 34 / 6 \%$ & Unclear \\
\hline CMJ & $2.0(-2.6 ; 6.7)$ & $0.22(-0.30 ; 0.75)$ & $53 / 38 / 9 \%$ & Unclear \\
\hline COD & $1.6(-0.4 ; 3.5)$ & $0.38(-0.09 ; 0.86)$ & $75 / 22 / 2 \%$ & Likely \\
\hline RSCODb & $1.6(0.5 ; 2.8)$ & $0.58(0.16 ; 1.00)$ & $93 / 6 / 0 \%$ & Likely \\
\hline RSCODt & $2.0(1.2 ; 2.7)$ & $0.99(0.60 ; 1.38)$ & $100 / 0 / 0 \%$ & Most Likely \\
\hline S-30 & $-0.9(-3.7 ; 1.7)$ & $-0.15(-0.60 ; 0.29)$ & $9 / 49 / 42 \%$ & Unclear \\
\hline \multicolumn{5}{|c|}{ WU vs. $60 \%-1$ RM } \\
\hline H3Jd & $-4.3(-5.8 ;-2.7)$ & $-0.77(-1.06 ;-0.48)$ & 0/0/100\% & Most Likely \\
\hline H3Jnd & $-3.8(-6.0 ;-1.6)$ & $-0.91(-1.46 ;-0.37)$ & 0/2/98\% & Very Likely \\
\hline SJ & $-8.0(-11.8 ;-4.0)$ & $-0.60(-0.91 ;-0.30)$ & 0/2/98\% & Very Likely \\
\hline CMJ & $-3.5(-5.0 ;-1.9)$ & $-0.40(-0.59 ;-0.22)$ & 0/3/97\% & Very Likely \\
\hline COD & $-2.0(-3.3 ;-0.7)$ & $-0.48(-0.80 ;-0.17)$ & 0/6/93\% & Likely \\
\hline RSCODb & $-2.1(-3.7 ;-0.4)$ & $-0.72(-1.28 ;-0.15)$ & $1 / 6 / 94 \%$ & Likely \\
\hline RSCODt & $0.5(-0.1 ; 1.1)$ & $0.26(-0.03 ; 0.56)$ & $65 / 34 / 1 \%$ & Possibly \\
\hline S-30 & $0.2(0.1 ; 0.4)$ & 0.04 (0.01; 0.07$)$ & 0/100/0\% & Most Likely trivial \\
\hline
\end{tabular}

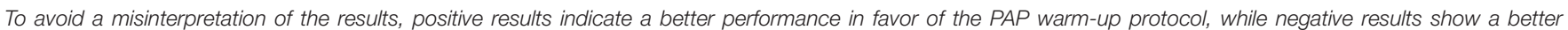

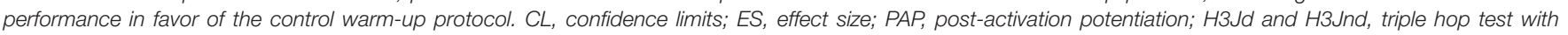

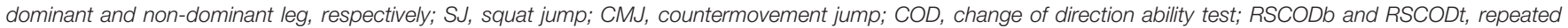

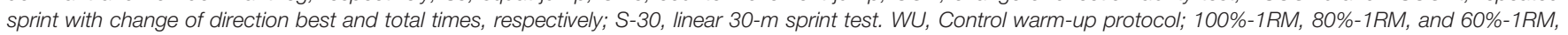
post-activation potentiation warm-up protocols at 60, 80 , and $100 \%$ of one repetition maximum in half-squat.

given the limited literature related to COD performance and PAP (Lockie et al., 2017). However, two previous studies observed improvements in COD performance after warm-up actions that included loaded exercises (Zois et al., 2011; Sole et al., 2013), and the improvement may be related to acute increase of reactive strength (Sole et al., 2013). Reactive strength is the ability to quickly change from the eccentric to the concentric phase during a stretch-shortening cycle muscle action (Young et al., 1998). In this sense, a greater reactive strength may help to improve the ability to perform sudden stops and to accelerate from there (Spiteri et al., 2013), hence improving COD speed (Sheppard and Young, 2006). Of note, a greater PAP effect (i.e., greater COD performance) was observed after the $80 \%-1 \mathrm{RM}$ versus the $60 \%-1 \mathrm{RM}$, since the load used in the $80 \%-1 \mathrm{RM}$ may help to maximize the acceleration phase of the COD action (McBride et al., 2005), implicating a better use of the stretch-shortening cycle in the deceleration-acceleration transition of the COD movement. However, the 100\%-1RM did not maximize COD performance. In this sense, the PAP effect may not proportionally depend on the load used (i.e., the higher the better), but other factors also may modulate the effect, such as the muscle fiber type, athletes' performance level, exercise type, time interval between the conditioning stimulus and the performance testing, among others (Sanchez-Sanchez et al., 2018). In fact, it has been suggested that there are PAP responders that may benefit from exercises designed to induce PAP, whereas others may not respond (Evetovich et al., 2015).

Regarding the RSCOD test, likely and most likely improvements were found in RSCODb and RSCODt with $80 \%-1 \mathrm{RM}$ in comparison to WU, respectively. Improvements in RSCOD performance after loaded back half-squat PAP protocols have been previously observed in elite male handball (Okuno et al., 2013) and soccer players (Sanchez-Sanchez et al., 2018). The 80\%-1RM may improve neuromuscular capacity (Hodgson et al., 2005), allowing an increase in athlete's power (Tillin and Bishop, 2009), thus better ability to repeat sprints (Glaister, 2005). Although warm-ups delivered to induce PAP may increase RSCOD performance, the load used must be applied with caution. Heavy loads (>90\% 1RM), with recovery times of 8 -minutes, may allow improvements in the total time and sprint time in a repeated sprint test (Low et al., 2015). However, the use of a heavier load (i.e., 100\%-1RM protocol) 
TABLE 3 | Comparisons of soccer player's physical performance after three different warm-up conditions.

\begin{tabular}{|c|c|c|c|c|}
\hline & $\%(C L 90 \%)$ & ES (CL90\%) & Chances & Outcome \\
\hline \multicolumn{5}{|c|}{$100 \%-1$ RM vs. $80 \%-1$ RM } \\
\hline H3Jd & $5.1(3.7 ; 6.5)$ & $0.79(0.58 ; 1.00)$ & $100 / 0 / 0 \%$ & Most Likely \\
\hline H3Jnd & $2.5(1.1 ; 3.9)$ & $0.35(0.15 ; 0.55)$ & $90 / 10 / 0 \%$ & Likely \\
\hline SJ & $5.9(3.3 ; 8.7)$ & $0.40(0.22 ; 0.57)$ & $96 / 4 / 0 \%$ & Very Likely \\
\hline CMJ & $5.7(2.5 ; 9.1)$ & $0.38(0.16 ; 0.59)$ & $92 / 8 / 0 \%$ & Likely \\
\hline COD & $3.6(1.4 ; 5.7)$ & $0.65(0.25 ; 1.04)$ & $97 / 3 / 0 \%$ & Very Likely \\
\hline RSCODb & $4.5(3.6 ; 5.4)$ & $2.15(1.70 ; 2.59)$ & $100 / 0 / 0 \%$ & Most Likely \\
\hline RSCODt & $2.3(1.6 ; 2.9)$ & $1.21(0.84 ; 1.57)$ & $100 / 0 / 0 \%$ & Most Likely \\
\hline S-30 & $0.8(-2.1 ; 3.5)$ & $0.13(-0.36 ; 0.63)$ & $41 / 47 / 12 \%$ & Possibly \\
\hline \multicolumn{5}{|c|}{$100 \%-1$ RM vs. $60 \%-1$ RM } \\
\hline H3Jd & $-2.3(-5.6 ; 1.1)$ & $-0.37(-0.92 ; 0.17)$ & $4 / 24 / 71 \%$ & Possibly \\
\hline H3Jnd & $-3.5(-6.8 ;-0.1)$ & $-0.51(-1.01 ;-0.01)$ & $1 / 13 / 86 \%$ & Likely \\
\hline SJ & $-6.1(-11.8 ; 0.0)$ & $-0.43(-0.86 ; 0.00)$ & $1 / 16 / 83 \%$ & Likely \\
\hline CMJ & $0.1(-5.7 ; 6.2)$ & $0.01(-0.39 ; 0.40)$ & $20 / 62 / 18 \%$ & Possibly trivial \\
\hline COD & $0.1(-2.4 ; 2.5)$ & $0.01(-0.43 ; 0.46)$ & $23 / 57 / 20 \%$ & Possibly trivial \\
\hline RSCODb & $0.9(-0.9 ; 2.7)$ & $0.41(-0.44 ; 1.26)$ & $67 / 22 / 11 \%$ & Possibly \\
\hline RSCODt & $0.8(0.2 ; 1.5)$ & $0.43(0.08 ; 0.78)$ & $87 / 12 / 0 \%$ & Likely \\
\hline S-30 & $1.9(1.4 ; 2.4)$ & $0.34(0.25 ; 0.43)$ & $99 / 1 / 0 \%$ & Very Likely \\
\hline \multicolumn{5}{|c|}{$60 \%-1 R M$ vs. $80 \%-1 R M$} \\
\hline H3Jd & $7.6(4.3 ; 10.9)$ & $0.88(0.51 ; 1.24)$ & $100 / 0 / 0 \%$ & Most Likely \\
\hline H3Jnd & $6.2(3.1 ; 9.4)$ & $0.90(0.45 ; 1.34)$ & $99 / 1 / 0 \%$ & Very Likely \\
\hline SJ & 12.9 (5.8; 20.3) & $0.72(0.34 ; 1.11)$ & $98 / 2 / 0 \%$ & Very Likely \\
\hline CMJ & $5.6(0.3 ; 11.2)$ & $0.61(0.04 ; 1.18)$ & $89 / 10 / 1 \%$ & Likely \\
\hline COD & $3.6(1.5 ; 5.9)$ & $0.87(0.36 ; 1.39)$ & $98 / 2 / 0 \%$ & Very Likely \\
\hline RSCODb & $3.8(1.6 ; 6.0)$ & $1.46(0.64 ; 2.29)$ & $99 / 1 / 0 \%$ & Very Likely \\
\hline RSCODt & $1.5(0.6 ; 2.4)$ & 0.69 (0.27; 1.10) & $97 / 3 / 0 \%$ & Very Likely \\
\hline S-30 & $-1.2(-3.8 ; 1.6)$ & $-0.16(-0.53 ; 0.21)$ & $5 / 52 / 42 \%$ & Possibly \\
\hline
\end{tabular}

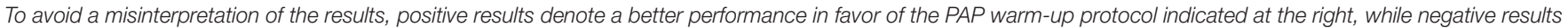

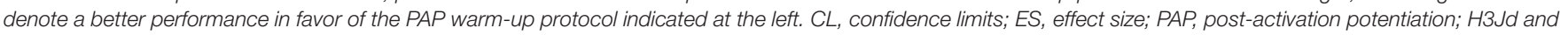

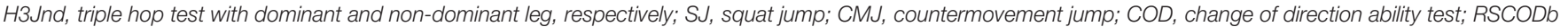

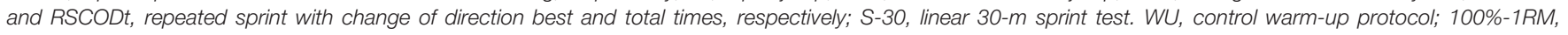
80\%-1RM, and 60\%-1RM, PAP warm-up protocols at 60, 80, and 100\% of one repetition maximum in half-squat.

may induce most likely poorer performance in the RSCODb in comparison to a WU, even when 8-minutes of rest are allowed. Although potentiation and fatigue coexist, the 100\%-1RM protocol may have induced fatigue to a greater extent than its PAP effect, potentially due to decreased release of calcium from the sarcoplasmic reticulum, leading to reduced calcium concentration in the myoplasm (Rassier and MacIntosh, 2000). In this sense, to optimally induce PAP, the load used must be selected accurately in male soccer players (Hammami et al., 2018). Although previous studies analyzed the effect of PAP warm-ups on RSCOD (Sanchez-Sanchez et al., 2018), this is the first study that compared the effects of three different loaded protocols on RSCOD performance, a key fitness specific-trait for soccer (Schimpchen et al., 2015), which determines match physical performance (Rampinini et al., 2007) and differentiates between competitive levels (Rampinini et al., 2009). Therefore, current findings may help practitioners to optimally prepare players before a match.

No changes in S-30 performance was observed after the $60 \%$ $1 \mathrm{RM}$ and the $80 \%-1 \mathrm{RM}$ compared to WU. No effects in sprint time after heavy loaded squat PAP protocols have been previously observed in soccer players (Tillin and Cooke, 2009). In contrast, the positive effects of heavy-load squats was obtained at distances of 10 to 40-m (McBride et al., 2005; Chatzopoulos et al., 2007; Rahimi, 2014). The mechanisms that underlie the effects of PAP warm-ups using loaded exercises on sprint performance have not been clarified (McBride et al., 2005). Maximal sprint velocity (i.e., distances $>30 \mathrm{~m}$ ) may depend on the force of the extensor muscles of the hip in order to re-incorporate the leg in the swing phase and thus maintain an adequate stride length (Weyand et al., 2000). Although the muscle force may be increased through a PAP warm-up, inducing an increased muscle stiffness (Moir et al., 2009), based on previous research, it was speculated that the PAP should be related to the volume of the pre-load (Evetovich et al., 2015). The PAP has traditionally been induced through the use of multiple sets of heavy isotonic resistance exercise (Wilson et al., 2013), but in this study only one set of 60\%-1RM and $80 \%-1$ RM was applied. From a practical perspective, given the timeframe separating the end of the warm-up and the start of a soccer match, there is no time to include multiple sets (Russell et al., 2014). On the other hand, a likely poorer performance after $100 \%-1 \mathrm{RM}$ compared to $\mathrm{WU}$ was observed. It is possible that 
the recovery time used in the present study (i.e., 5-minutes) was not enough to elicit a significant PAP effect after the 100\%-1RM in some players. Considering that fatigue and potentiation Coexist (Rassier and MacIntosh, 2000), shorter rest intervals may increase fatigue (Tillin and Cooke, 2009). In this sense, $<4 \mathrm{~min}$ of rest after a PAP warm-up of 5-RM did not induce an increase in sprint performance (Tillin and Cooke, 2009). However, $5 \mathrm{~min}$ of rest after a PAP warm-up, as used in the current study, seems to be adequate in order to reload phosphocreatine stores (Chatzopoulos et al., 2007). Still, this did not explain the lack of improvements or even poorer sprinting capability after the $100 \%$ 1RM strategy used in the current study. It has been suggested that in order to improve sprinting performance, the potential effect of heavier PAP protocols, including load scheme and rest time, should be prescribed on an individual basis (Mola et al., 2014).

Using an $80 \%-1 \mathrm{RM}$ protocol could be an effective strategy to enhance the physical performance of elite male soccer player, with potential implications for a better performance, especially at the beginning of games. Coaches should carefully consider the recovery time between the PAP application and the start of a match in order to reduce the risk of fatigue. Moreover, although in the current study an $80 \%-1 \mathrm{RM}$ protocol induced greater mean improvements in the physical performance of soccer players, PAP protocols should be elaborated considering athlete's individual characteristics.

Potential limitations of the current study are related to the lack of physiological and biochemical measurements, in order to further understand the underlying factors related with the observed PAP phenomenon. The limited number of subjects involved in the current study could be recognized as an additional limitation, in line with the use of magnitude-based inferences (MBI). Such statistical approach has been criticized as may induce a greater risk of type I error (Sainani, 2018). On the other side, its use have been strongly supported in sport science studies (Batterham and Hopkins, 2019). Future studies should strive to elucidate if current results may be transferred to competition scenarios, analyzing performance indices during a match, such as

\section{REFERENCES}

Barnes, K. R., Hopkins, W. G., McGuigan, M. R., and Kilding, A. E. (2015). Warmup with a weighted vest improves running performance via leg stiffness and running economy. J. Sci. Med. Sport 18, 103-108. doi: 10.1016/j.jsams.2013. 12.005

Batterham, A., and Hopkins, W. (2019). The problems with "The Problem with 'Magnitude-Based Inference”'. Med. Sci. Sports Exerc. 51:599.

Chatzopoulos, D. E., Michailidis, C. J., Giannakos, A. K., Alexiou, K. C., Patikas, D. A., Antonopoulos, C. B., et al. (2007). Postactivation potentiation effects after heavy resistance exercise on running speed. J. Strength Cond. Res. 21, 1278-1281. doi: 10.1519\%2F00124278-200711000-00051

Evetovich, T. K., Conley, D. S., and McCawley, P. F. (2015). Postactivation potentiation enhances upper- and lower-body athletic performance in collegiate male and remale athletes. J. Strength Cond. Res. 29, 336-342. doi: 10.1519/JSC. 0000000000000728

Glaister, M. (2005). Multiple sprint work: physiological responses, mechanisms of fatigue and the influence of aerobic fitness. Sport Med. 35, 757-777. doi: 10.2165\%2F00007256-200535090-00003

González-Mohíno, F., Martín, R., Juárez Santos-García, D., Fidel, P. A., de AsísFernández, F., Yustres, I., et al. (2018). Effects of high-intensity warm-ups on covered distance at high-intensity, accelerations, among others short-term high-intensity actions. In addition, future studies may analyse the potential interfering effect (if any) of repeated physical fitness test (i.e., H3JD, SJ) performed on the same testing session and its role on PAP after warm-ups using different 1RM back-squat intensities.

\section{CONCLUSION}

In conclusion, a moderate intensity (i.e., 80\%-1RM back squat) may induce greater improvements (i.e., PAP effect) in jumping, repeated COD speed and non-repeated COD speed in elite level male soccer players when compared to low (60\%-1RM) and high (100\%-1RM) intensity warm-up protocols.

\section{ETHICS STATEMENT}

This study was carried out in accordance with the recommendations of "Ethics Committee, Pontifical University of Salamanca" with written informed consent from all subjects. All subjects gave written informed consent in accordance with the Declaration of Helsinki. The protocol was approved by the "Ethics Committee, Pontifical University of Salamanca (Annex III, Act 13/2/2019)."

\section{AUTHOR CONTRIBUTIONS}

CP, RR-C, and JS-S designed the work. CP, DH, and JS-S acquired the data. CP, FN, RR-C, JS-S, and OG-S analyzed and interpreted of data. All authors drafted the manuscript, critically revised the manuscript and approved the final version of the manuscript to be published. CP, RR-C, FN, and JS-S agreed to be accountable for all aspects of the work in ensuring that questions related to the accuracy or integrity of any part of the work were appropriately investigated and resolved.

running performance. Int. J. Sports Med. 39, 426-432. doi: 10.1055/s-0044102132

Gouvêa, A. L., Fernandes, I. A., César, E. P., Silva, W. A. B., and Gomes, P. S. C. (2013). The effects of rest intervals on jumping performance: a meta-analysis on post-activation potentiation studies. J. Sports Sci. 31, 459-467. doi: 10.1080/ 02640414.2012.738924

Hamada, T., Sale, D. G., MacDougall, J. D., and Tarnopolsky, M. A. (2000). Postactivation potentiation, fiber type, and twitch contraction time in human knee extensor muscles. J. Appl. Physiol. 88, 2131-2137. doi: 10.1152\%2Fjappl. 2000.88.6.2131

Hammami, A., Zois, J., Slimani, M., Russel, M., and Bouhlel, E. (2018). The efficacy, and characteristics, of warm-up and re-warm-up practices in soccer players: a systematic review. J. Sports Med. Phys. Fitness 58, 135-149. doi: 10.23736/ S0022-4707.16.06806-7

Hodgson, M., Docherty, D., and Robbins, D. (2005). Post-activation potentiation: underlying physiology and implications for motor performance. Sport Med. 35, 585-595. doi: 10.2165\%2F00007256-20053507000004

Hopkins, W. G., Marshall, S. W., Batterham, A. M., and Hanin, J. (2009). Progressive statistics for studies in sports medicine and exercise science. Med. Sci. Sports Exerc. 41, 3-13. 
Iaia, F. M., Rampinini, E., and Bangsbo, J. (2009). High-intensity training in football. Int. J. Sports Physiol. Perform. 4, 291-306.

Lockie, R. G., Lazar, A., Davis, D. L., and Moreno, M. R. (2017). Effects of postactivation potentiation on linear and change-of-direction speed: analysis of the current literature and applications for the strength and conditioning coach. Strength Cond. J. 40, 75-91.

Low, D., Harsley, P., Shaw, M., and Peart, D. (2015). The effect of heavy resistance exercise on repeated sprint performance in youth athletes. J. Sports Sci. 33, 1028-1034. doi: 10.1080/02640414.2014.979857

Maulder, P., Cronin, J., Maulder, P., and Cronin, J. (2015). Horizontal and vertical jump assessment: reliability, symmetry, discriminative and predictive ability discriminative and predictive ability. Phys. Ther. Sport 6, 74-82. doi: $10.1016 \%$ 2Fj.ptsp.2005.01.001

McBride, J. M., Nimphius, S., and Erickson, T. M. (2005). The acute effects of heavy-load squats and loaded countermovement jumps on sprint performance. J. Strength Cond. Res. 19, 893-897. doi: 10.1519\%2F00124278-20051100000029

Moir, G. L., Dale, J. R., and Dietrich, W. W. (2009). The acute effects of heavy back squats on machanical variables during a series of bilateral hops. J. Strength Cond. Res. 23, 1118-1124. doi: 10.1519/JSC.0b013e31819166c2

Mola, J. N., Bruce-Low, S. S., and Burnet, S. J. (2014). Optimal recovery time for postactivation potentiation in professional soccer players. J. Strength Cond. Res. 28, 1529-1537. doi: 10.1519/JSC.0000000000000313

Noyes, F. R., Barber, S. D., and Mangine, R. E. (1991). Abnormal lower limb symmetry determined by functional hop tests after anterior cruciate ligament rupture. Am. J. Sports Med. 19, 513-518. doi: 10.1177\%2F036354659101900518

Okuno, N. M., Tricoli, V., Silva, S. B., Bertuzzi, R., Moreira, A., and Kiss, M. (2013). Postactivation potentiation on repeated-sprint ability in elite handball players. J. Strength Cond. Res. 27, 662-668. doi: 10.1519/JSC.0b013e31825bb582

Rahimi, R. (2014). The acute effects of heavy versus light-load squats on sprint performance. Phys. Educ. Sport 5, 163-169.

Rampinini, E., Couts, A. J., Castagna, C., Sassi, R., and Impellizzeri, F. M. (2007). Variation in top level soccer match performance. Int. J. Sports Med. 28, 10181024. doi: 10.1055\%2Fs-2007-965158

Rampinini, E., Sassi, A., Morelli, A., Mazzoni, S., Fanchini, M., and Coutts, A. J. (2009). Repeated-sprint ability in professional and amateur soccer players. Appl. Physiol. Nutr. Metab. 34, 1048-1054. doi: 10.1139/H09-111

Rassier, D. E., and MacIntosh, B. R. (2000). Coexistence of potentiation and fatigue in skeletal muscle. Braz. J. Med. Biol. Res. 33, 499-508. doi: 10.1590\%2Fs0100879x2000000500003

Russell, M., Cook, C. J., and Kilduff, L. P. (2014). "Match day strategies to enhance the physical and technical performance of rugby players," in The Science of Rugby, eds C. Twist and P. Worsfold (London: Routledge), 97-114.

Saez-Saez de Villarreal, E., Gonzalez-Badillo, J. J., and Izquierdo, M. (2007). Optimal warm-up stimuli of muscle activation to enhance short and long-term acute jumping performance. Eur. J. Appl. Physiol. 100, 393-401. doi: 10.1007\% 2Fs00421-007-0440-9

Sainani, K. L. (2018). The problem with "Magnitude-based Inference". Med. Sci. Sports Exerc. 50, 2166-2176. doi: 10.1249\%2Fmss.0000000000001645

Sale, D. (2004). Postactivation potentiation: role in performance. Br. J. Sports Med. 38, 386-387. doi: 10.1136\%2Fbjsm.2002.003392

Sanchez-Sanchez, J., Rodríguez-Fernández, A., Petisco, C., Ramirez-Campillo, R., and Nakamura, F. Y. (2018). Effects of different post-activation potentiation warm-ups on repeated sprint ability in soccer players from different competitive levels. J. Hum. Kinet. 61, 189-197. doi: 10.1515/hukin-2017-0131

Sassi, R. H., Dardouri, W., Yahmed, M. H., Gmada, N., Mahfoudhi, M. E., and Gharbi, Z. (2009). Relative and absolute reliability of a modified agility T-test and its relationship with vertical jump and straight sprint. J. Strength Cond. Res. 23, 1644-1651. doi: 10.1519/JSC.0b013e3181b425d2

Schimpchen, J., Skorski, S., Nopp, S., and Meyer, T. (2015). Are "classical” tests of repeated-sprint ability in football externally valid? A new approach to determine in-game sprinting behaviour in elite football players. J. Sports Sci. 34, 519-526. doi: 10.1080/02640414.2015.1112023

Seitz, L. B., and Haff, G. G. (2016). Factors modulating post-activation potentiation of jump, sprint, throw, and upper-body ballistic performances: a systematic review with meta-analysis. Sport Med. 46, 231-240. doi: 10.1007/s40279-0150415-7

Sheppard, J. M., and Young, W. B. (2006). Agility literature review: classifications, training and testing. J. Sports Sci. 24, 919-932. doi: 10.1080\%2F02640410500457109

Sole, C. J., Moir, G. L., Davis, S. E., and Witmer, C. A. (2013). Mechanical analysis of the acute effects of a heavy resistance exercise warm-up on agility performance in court-sport athletes. J. Hum. Kinet. 39, 147-156. doi: 10.2478/hukin-20130077

Spiteri, T., Cochrane, J. L., Hart, N. H., Haff, G. G., and Nimphius, S. (2013). Effect of strength on plant foot kinetics and kinematics during a change of direction task. Eur. J. Sport Sci. 13, 646-652. doi: 10.1080/17461391.2013.774053

Stølen, T., Chamari, K., Castagna, C., and Wisløff, U. (2005). Physiology of soccer. Sport Med. 35, 501-536.

Tillin, K. A., and Cooke, C. (2009). The effects of postactivation potentiation on sprint and jump performance of male academy soccer players. J. Strength Cond. Res. 23, 1960-1967. doi: 10.1519/JSC.0b013e3181b8666e

Tillin, N. A., and Bishop, D. (2009). Factors modulating post-activation potentiation and its effect on performance of subsequent explosive activities. Sport Med. 39, 147-166. doi: 10.2165/00007256-200939020-00004

Turner, A. P., Bellhouse, S., Kilduff, L. P., and Russell, M. (2015). Post-activation potentiation of sprint acceleration performance using plyometric exercise. J. Strength Cond. Res. 29, 343-350. doi: 10.1519\%2Fjsc.0000000000000647

Weyand, P. G., Sternlight, D. B., Bellizzi, M. J., and Wright, S. (2000). Faster top running speeds are achieved with greater ground forces not more rapid leg movements. J. Appl. Physiol. 89, 1991-1999. doi: 10.1152\%2Fjappl.2000.89.5. 1991

Wilson, J. M., Duncan, N. M., Marin, P. J., Brown, L. E., Loenneke, J. P., Wilson, S., et al. (2013). Meta-Analysis of postactivation potentiation and power: effects of conditioning activity, volume, gender, rest periods, and training status. J. Strength Cond. Res. 27, 854-859. doi: 10.1519/JSC.0b013e31825c2bdb

Young, W. B., Jenner, A., and Griffiths, K. (1998). Acute enhancement of power performance from heavy load squats. J. Strength Cond. Res. 12, 82-84. doi: 10.1519\%2F00124278-199805000-00004

Zois, J., Bishop, D. J., Ball, K., and Aughey, R. J. (2011). High-intensity warm-ups elicit superior performance to a current soccer warm-up routine. J. Sci. Med. Sport 14, 522-528. doi: 10.1016/j.jsams.2011.03.012

Conflict of Interest Statement: The authors declare that the research was conducted in the absence of any commercial or financial relationships that could be construed as a potential conflict of interest.

Copyright (C) 2019 Petisco, Ramirez-Campillo, Hernández, Gonzalo-Skok, Nakamura and Sanchez-Sanchez. This is an open-access article distributed under the terms of the Creative Commons Attribution License (CC BY). The use, distribution or reproduction in other forums is permitted, provided the original author(s) and the copyright owner(s) are credited and that the original publication in this journal is cited, in accordance with accepted academic practice. No use, distribution or reproduction is permitted which does not comply with these terms. 